\title{
Panitumumab: the evidence for its use in the treatment of metastatic colorectal cancer
}

This article was published in the following Dove Press journal:

Core Evidence

25 September 2010

Number of times this article has been viewed

Rossana Berardi'

Azzurra Onofri ${ }^{2}$

Mirco Pistelli ${ }^{2}$

Elena Maccaroni ${ }^{2}$

Mario Scartozzi'

Chiara Pierantoni'

Stefano Cascinu'

'Clinica di Oncologia Medica, Università Politecnica delle Marche, Ospedali Riuniti Umberto I-GM Lancisi-G Salesi di Ancona, Italy; ${ }^{2}$ Scuola di Specializzazione in Oncologia Medica, Università Politecnica delle Marche, Ancona, Italy
Correspondence: Rossana Berardi Medical Oncology Unit, Università Politecnica delle Marche, Azienda Ospedaliero-Universitaria Ospedali Riuniti Umberto I, GM Lancisi,

G Salesi di Ancona, Via Conca

71, 60020, Ancona, Italy

$\mathrm{Tel}+390715965715$

Fax +39 07I 5965053

Email r.berardi@univpm.it
Abstract: Panitumumab is the first fully human monoclonal antibody to Epidermal Growth Factor Receptor (EGFR) to enter clinical trials for the treatment of solid tumors. The anti-tumor activity of panitumumab has been tested in vitro and in vivo, and inhibition of tumor growth has been observed in numerous cancer models, particularly lung, kidney and colorectal (CRC). Preclinical and clinical studies have established a role for panitumumab in metastatic colorectal cancer ( $\mathrm{mCRC}$ ) refractory to multiple chemotherapeutic regimens. Based on these encouraging findings, panitumumab was approved by the US Food and Drug Administration for the treatment of patients with epidermal growth factor receptor-expressing mCRC refractory to fluoropyrimidine-, oxaliplatin-, and/or irinotecan-containing chemotherapeutic regimens. The improvement in progression free survival (PFS) and response rate (RR) produced by panitumumab monotherapy was significantly greater in patients with non mutated (wild-type) K-RAS than in those with mutant K-RAS. Therefore implementing routine K-RAS screening and limiting the use of EGFR inhibitors to patients with wild-type K-RAS appears the better strategy for select only the patients who could benefit from the therapy with panitumumab and also may have the potential for cost savings. The purpose of this review was to evaluate the patient-related, disease-related and economic-related evidence for the use of panitumumab in the treatment of metastatic colorectal cancer in clinical practice.

Keywords: colorectal cancer, EGFR; K-RAS, panitumumab

\begin{tabular}{|c|c|c|}
\hline Outcome measure & Evidence & Implications \\
\hline \multicolumn{3}{|l|}{$\begin{array}{l}\text { Disease-oriented } \\
\text { evidence }\end{array}$} \\
\hline Phase I-II studies & $\begin{array}{l}\text { Panitumumab was well tolerated, } \\
\text { and no human anti-human } \\
\text { antibody formation or infusion-related } \\
\text { reactions were observed. } \\
\text { Moreover, the use of panitumumab } \\
\text { increased overall response } \\
\text { rate and seemed to improve } \\
\text { PFS and OS. }\end{array}$ & $\begin{array}{l}\text { Panitumumab was evaluated in } \\
\text { phase III trials in patients with } \\
\text { relapsed or refractory metastatic } \\
\text { CRC. }\end{array}$ \\
\hline Phase III & $\begin{array}{l}\text { Panitumumab significantly } \\
\text { improved overall response rate, } \\
\text { PFR and OS in } \mathrm{mCRC} \text { pretreated } \\
\text { patients. }\end{array}$ & $\begin{array}{l}\text { Panitumumab monotherapy } \\
\text { received FDA approval for the } \\
\text { treatment of metastatic colorectal } \\
\text { cancer with disease progression } \\
\text { while receiving or after receiving } \\
\text { fluoropyrimidine, oxaliplatin, and } \\
\text { irinotecan chemotherapy regimens. }\end{array}$ \\
\hline
\end{tabular}




\begin{tabular}{|lll|}
\hline $\begin{array}{l}\text { (Continued) } \\
\text { Patient-oriented evidence }\end{array}$ & \\
\hline K-RAS & $\begin{array}{l}\text { Clinical efficacy of panitumumab } \\
\text { therapy is restricted to patients with } \\
\text { wild-type K-RAS tumors. There was } \\
\text { no evidence of benefit in patients with } \\
\text { mutated K-RAS tumors. }\end{array}$ & $\begin{array}{l}\text { K-RAS genotyping of tumors should } \\
\text { be strongly considered to select } \\
\text { patients being treated with } \\
\text { panitumumab. }\end{array}$ \\
Skin Toxicity & $\begin{array}{l}\text { The development of skin toxicity } \\
\text { during panitumumab monotherapy } \\
\text { has been significantly linked }\end{array}$ & $\begin{array}{l}\text { Skin toxicity cannot be used to } \\
\text { select patients and it could be useful } \\
\text { in the clinical practice to identify }\end{array}$ \\
& $\begin{array}{l}\text { with higher response rate and longer } \\
\text { survival. }\end{array}$ & $\begin{array}{l}\text { patients who may derive greater } \\
\text { benefit from panitumumab }\end{array}$ \\
treatment.
\end{tabular}

\section{Introduction}

The most recent improvements in the treatment of mCRC have derived from the development of targeted therapy toward cell surface receptors and their associated intracellular second-messenger systems. Targeted therapy against tumors is an attractive therapeutic strategy in the treatment of human malignancies, complementing currently available chemotherapeutic agents and avoiding overlapping toxicities, as well as potentially improving clinical response rates and patient survival.

Recently, EGFR has been validated as a therapeutic target in several human tumors, including colorectal cancer (CRC).

In fact, overexpression or dysregulation of EGFR has been reported in several solid tumors and it is associated with tumor cell proliferation, invasion, distant metastasis, angiogenesis, antiapoptosis, and resistance to chemotherapy or radiation therapy. In CRC, EGFR is overexpressed in $60 \%-80 \%$ of tumors, and the extent of EGFR expression has been shown to positively correlate with a poor prognosis. ${ }^{1,2}$

The EGFR-targeted monoclonal antibodies block the interaction between a ligand and the extracellular binding domain of EGFR, inhibiting both phosphorylation and activation of EGFR-associated kinases (such as epidermal growth factor (EGF), transforming growth factor- $\alpha$ (TGF- $\alpha)$ ), causing internalization of the receptor, inhibition of cellular growth, induction of apoptosis, and decreased production of growth factors (such as proinflammatory cytokines, vascular endothelial growth factor (VEGF)). ${ }^{3,4}$

Both monoclonal antibodies and small molecules inhibitors of the tyrosine-kinase of EGFR have been evaluated in the treatment of solid tumors, including CRC, non-small cell lung cancer, squamous cell carcinoma of the head and neck. ${ }^{5}$ Currently, two monoclonal antibodies targeting the EGFR, cetuximab and panitumumab, are commercially available for the treatment of mCRC.

\section{Panitumumab}

Panitumumab, formerly known as ABX-EGF, is a fully human IgG2 monoclonal antibody targeting EGFR and developed using XenoMouse (Abgenix, Fremont, CA, USA) technology. In vitro, panitumumab has been found to have high binding affinity to EGFR, competitively blocking binding of EGF and TGF- $\alpha$ to the receptor and leading to internalization of the receptor-antibody complex. This prevents ligand-induced EGFR-tyrosine autophosphorylation and subsequent activation of key downstream signaling molecules involved in carcinogenesis. This leads to antitumor effects by promoting apoptosis and inhibiting cell proliferation, growth and angiogenesis. ${ }^{6,7}$

Since it is the first fully human monoclonal antibody, the risk of hypersensitivity reactions with panitumumab is reduced, and this may be important for long-term administration. ${ }^{8}$ 


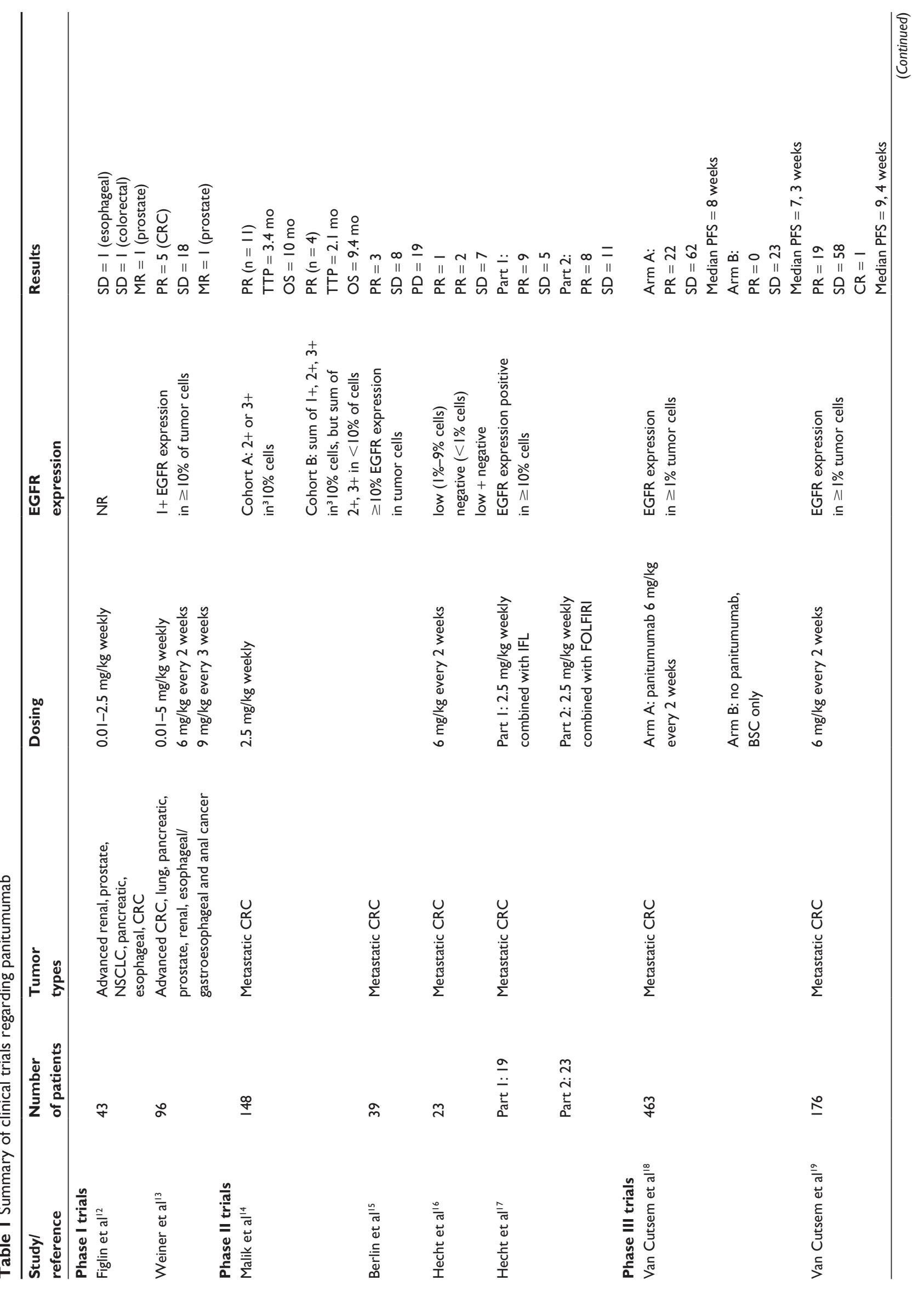




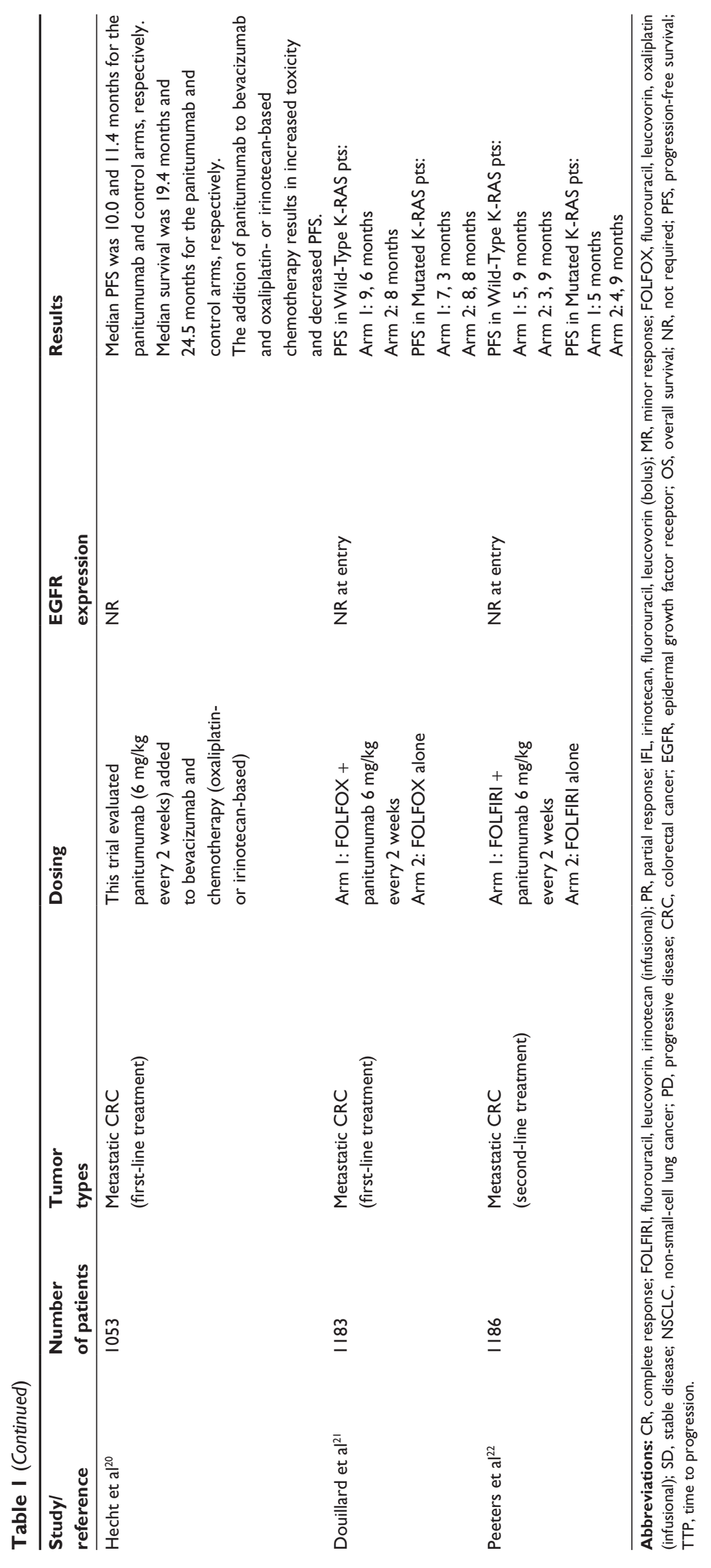


Panitumumab induces cell-cycle arrest in the G0-G1 interphase, whereas cetuximab causes arrest in the G1 phase. Unlike cetuximab, panitumumab does not induce antibody-dependent cellular cytotoxicity. ${ }^{9,10}$

Panitumumab was initially studied as a single agent in previously treated patients with metastatic colorectal cancer and showed promising antitumor responses and minimal adverse effects, offering an alternative to cetuximab as a second-or third-line treatment option for patients with metastatic colorectal cancer who have failed prior therapies.

\section{Disease-oriented evidence Phase I and II studies}

Panitumumab has been evaluated in clinical trials both as monotherapy and in combination with other agents for the treatment of solid tumors, including colorectal and kidney cancer (Table 1). ${ }^{11}$

A phase I trial by Figlin et $\mathrm{al}^{12}$ using doses ranging from $0.1 \mathrm{mg} / \mathrm{kg}$ up to $2.5 \mathrm{mg} / \mathrm{kg}$ was performed in 43 patients with several tumor types (renal $=10$; prostate $=3$; nonsmall-cell lung cancer $=7$; pancreatic $=3$; esophageal $=3$ and $\mathrm{CRC}=7$ ). Patients received up to 4 weekly doses, and those experiencing response or stable disease (SD) were eligible to continue to receive treatment every other week for 6 additional months or until disease progression. Biologic activity was seen even with low doses, including one patient with esophageal cancer treated with the lowest dose that had SD for 7 months. A partial response (PR) of 10 months was seen in one patient with CRC treated with $2.5 \mathrm{mg} / \mathrm{kg}$. The incidence of skin rash in patients receiving 2.0 or $2.5 \mathrm{mg} / \mathrm{kg}$ approached $100 \%$. Overall, panitumumab was well tolerated and no allergic reactions, infusion-related or serious adverse events were observed.

Weiner et $\mathrm{al}^{13}$ updated these data in with another phase I trial. 96 patients were enrolled and treated $(\mathrm{CRC}=39$, lung $=14$, pancreatic $=3$, prostate $=21$, renal $=15$, esophageal $=3$ and anal cancer $=1$ ). Sequential cohorts were enrolled to receive four infusions of panitumumab monotherapy at different dose levels ranging from 0.01 to $5.0 \mathrm{mg} / \mathrm{kg}$ once per week, $6.0 \mathrm{mg} / \mathrm{kg}$ every 2 weeks and $9.0 \mathrm{mg} / \mathrm{kg}$ every 3 weeks. Grade 3 or 4 related adverse events were noted in $10 \%$ of patients, with grade 3 skin-related effects being the most frequent ( $7 \%$ of patients). No maximally tolerated dose was reached and no infusionrelated reactions were observed. Furthermore, five of the 39 CRC patients achieved a PR.

After the favourable response rate observed among patients with $\mathrm{CRC}$ participating in these studies, panitumumab was evaluated in phase II trials in patients with relapsed or refractory metastatic CRC (Table 1).

One study included patients who had failed therapy with a fluoropyrimidine (with or without leucovorin) and either irinotecan or oxaliplatin, or both. ${ }^{14}$ Assessment based on level of EGFR expression was also carried out and patients were enrolled into 2 cohorts. Patient cohorts were determined by levels of EGFR expression. Cohort A $(n=105)$ consisted of patients with $2+$ or $3+$ EGFR over-expression in $\geq 10 \%$ of tumor cells. Cohort B $(n=43)$ included patients with the sum of $1+, 2+$, and $3+$ EGFR staining found in $\geq 10 \%$ of tumor cells, but with the sum of $2+$ and $3+$ in $<10 \%$ of tumor cells. Patients received panitumumab $2.5 \mathrm{mg} / \mathrm{kg}$ weekly for 8 weekly cycles. Overall, 15 PR were reported: $11(10 \%)$ in cohort A and $4(9 \%)$ in cohort B. Median time to disease progression and median survival were 3.4 months and 10 months, respectively, for cohort A, and 2.1 months and 9.4 months, respectively, for cohort B.

In another phase II trial performed by Berlin et $\mathrm{al}^{15}$ panitumumab monotherapy was evaluated in patients with mCRC failing at least two previous regimens with a fluoropyrimidine, irinotecan, and oxaliplatin. Panitumumab was administered at $6 \mathrm{mg} / \mathrm{kg}$ intravenously every 2 weeks until disease progression occurred. Primary endpoints were: objective RR; response duration; PFS: and survival time. The secondary endpoint was tolerability. Assessment based on level of EGFR expression was also carried out, and all patients were required to have EGFR staining of $\geq 10 \%$ tumor cells on immunohistochemistry (IHC).

An interim analysis in May 2005 included 39 patients eligible for efficacy evaluation following $\geq 20$ weeks of treatment and 91 patients available for tolerability analysis after receiving at least 1 dose of panitumumab. At week 16, 3 (8\%) patients had PR, 8 patients $(21 \%)$ achieved SD, and 19 patients (49\%) experienced disease progression. Nine patients (22\%) were not assessable. Integument toxicities included skin (96\%), nail (30\%), chelitis (7\%), and hair (5\%). Eye toxicity occurred in $85 \%$ of patients; diarrhea, in $27 \%$ (3 with grade 3 ); and hypomagnesemia, in $12 \%$ (3 with grade 3 or grade 4 ). Grade 3 hypersensitivity reaction occurred in 1 patient and resolved with treatment. This study plans a total enrollment of 300 patients. ${ }^{15}$

Moreover, the results of another study showed, in patients with low or negative EGFR staining, a response rate of approximately $5 \% .^{16}$

Panitumumab was also evaluated in combination with fluorouracil/leucovorin and irinotecan (IFL or FOLFIRI 
regimens) for first-line treatment of metastatic colorectal cancer. Part 1 of the study included patients who received panitumumab with IFL $(n=19)$, and part 2 included patients who received panitumumab with FOLFIRI $(n=24)$. Eligibility criteria included no prior chemotherapy and EGFR positivity ( $\geq 10 \%$ ). Panitumumab was administered weekly at a dose of $2.5 \mathrm{mg} / \mathrm{kg}$ over one hour. Due to unacceptable toxicity in part 1 (58\% grade 3 or grade 4 diarrhea), the study was modified to evaluate panitumumab in combination with FOLFIRI (part 2).

No complete responses were observed. The investigators reported $9(47 \%)$ patients with PR and 5 (26\%) with SD for part 1 of the study. Among patients enrolled in part 2, there were $8(33 \%)$ with PR and $11(46 \%)$ with SD. Median PFS was 5.6 months and 10.9 months for parts 1 and 2, respectively. Median survival for patients enrolled in part 1 was 16.8 months. Survival data were not available for patients enrolled in part 2; however, 23 out of 24 patients were alive at the time of analysis. ${ }^{17}$

\section{Phase III studies}

Based on the encouraging clinical outcomes of the above mentioned phase II trials, a pivotal, randomized, controlled phase III trial conducted in Europe, Australia, and Canada was performed, in order to compare panitumumab $6 \mathrm{mg} / \mathrm{kg}$ intravenously every 2 weeks plus best supportive care (BSC) versus best supportive care alone. The aim of this study was to show the significant difference in PFS. A total of 463 patients were enrolled $(n=231$ receiving panitumumab plus $\mathrm{BSC}$ and $\mathrm{n}=232$ receiving $\mathrm{BSC}$ alone). Eligible patients had metastatic colorectal cancer ( $\geq 1 \%$ EGFR-positive tumor cells) and documented progressive disease during treatment or within 6 months of completing treatment with a fluoropyrimidine, irinotecan, and oxaliplatin.

Patients in the best supportive care group experiencing progressive disease could receive panitumumab in a crossover study. This study was designed to be able to detect a $33 \%$ difference in PFS, but the results far exceeded this with a risk reduction of $46 \%$, statistically significant with $P<0.000000001$. The overall response rate was $36 \%$ versus $10 \%$ (control) with a median duration of response of 17 weeks in the control arm. At 6 months, PFS was $18 \%$ versus $5 \%$ and this difference was maintained at 8 months: $10 \%$ versus $4 \%$. Although no difference has been noted in overall survival, this is likely to have been confounded by the fact that $75 \%$ of those on the best supportive care arm crossed over to panitumumab with impressive results. Of the
174 patients who crossed over to the treatment arm, there was a $9 \% \mathrm{PR}$ and $32 \%$ with SD.

The most common toxicities reported were skin toxicities, hypomagnesemia, and diarrhoea. Skin reaction occurred in $90 \%$ of patients receiving panitumumab and consistent with other reports, an association between severe rash and greater clinical efficacy was observed. As expected with fully human antibodies, panitumumab had a low frequency of infusion-related reactions and no antibody formation. An openlabel extension study showed similar results for those patients initially receiving best supportive care who later received panitumumab therapy. Based on these results, panitumumab monotherapy received FDA approval for the treatment of metastatic colorectal cancer with disease progression while receiving or after receiving fluoropyrimidine, oxaliplatin, and irinotecan chemotherapy regimens. ${ }^{18,19}$

The role of panitumumab in combination with antiangiogenic drugs has also been explored in a randomized phase III study (Panitumumab Advanced Colorectal Cancer Evaluation, (PACCE)). In this trial patients with $\mathrm{mCRC}$ were randomly assigned for first-line treatment within each chemotherapy cohort (823 patients oxaliplatin- and 230 irinotecan-based) to bevacizumab and chemotherapy with or without panitumumab $6 \mathrm{mg} / \mathrm{kg}$ every 2 weeks. Most patients received oxaliplatin-based chemotherapy. The primary end-point was PFS within the oxaliplatin cohort. The results of the study were negative, as the combination of panitumumab with bevacizumab and chemotherapy resulted in a decrease of PFS and in excessive toxicity, particularly diarrhoea, infections and pulmonary embolism. The results were consistent in both the oxaliplatin and irinotecan cohorts. Moreover, as demonstrated previously, the triple combination did not provide additional benefit in the K-RAS wild-type population treated with panitumumab. ${ }^{20}$

Recently, two large, randomized, phase III trials, were presented at 2009 Joint ECCO/ESMO Multidisciplinary Congress in Berlin, Germany. ${ }^{21,22}$

The PRIME trial was a multicenter, randomized, phase III study performed by Douillard et $\mathrm{al}^{21}$ in order to analyze the safety and efficacy of first-line treatment with panitumumab plus FOLFOX versus FOLFOX alone in mCRC according to K-RAS status.

Patients were randomized $1: 1$ to receive $6 \mathrm{mg} / \mathrm{kg}$ of panitumumab plus FOLFOX every 2 weeks (Arm 1) versus FOLFOX alone (Arm 2). The primary endpoint was PFS. The study randomized a total of 1183 patients, with 593 in Arm 1 and 590 in Arm 2. K-RAS results were obtained for $93 \%$ of patients: $60 \%$ were K-RAS wild-type and $40 \%$ were 


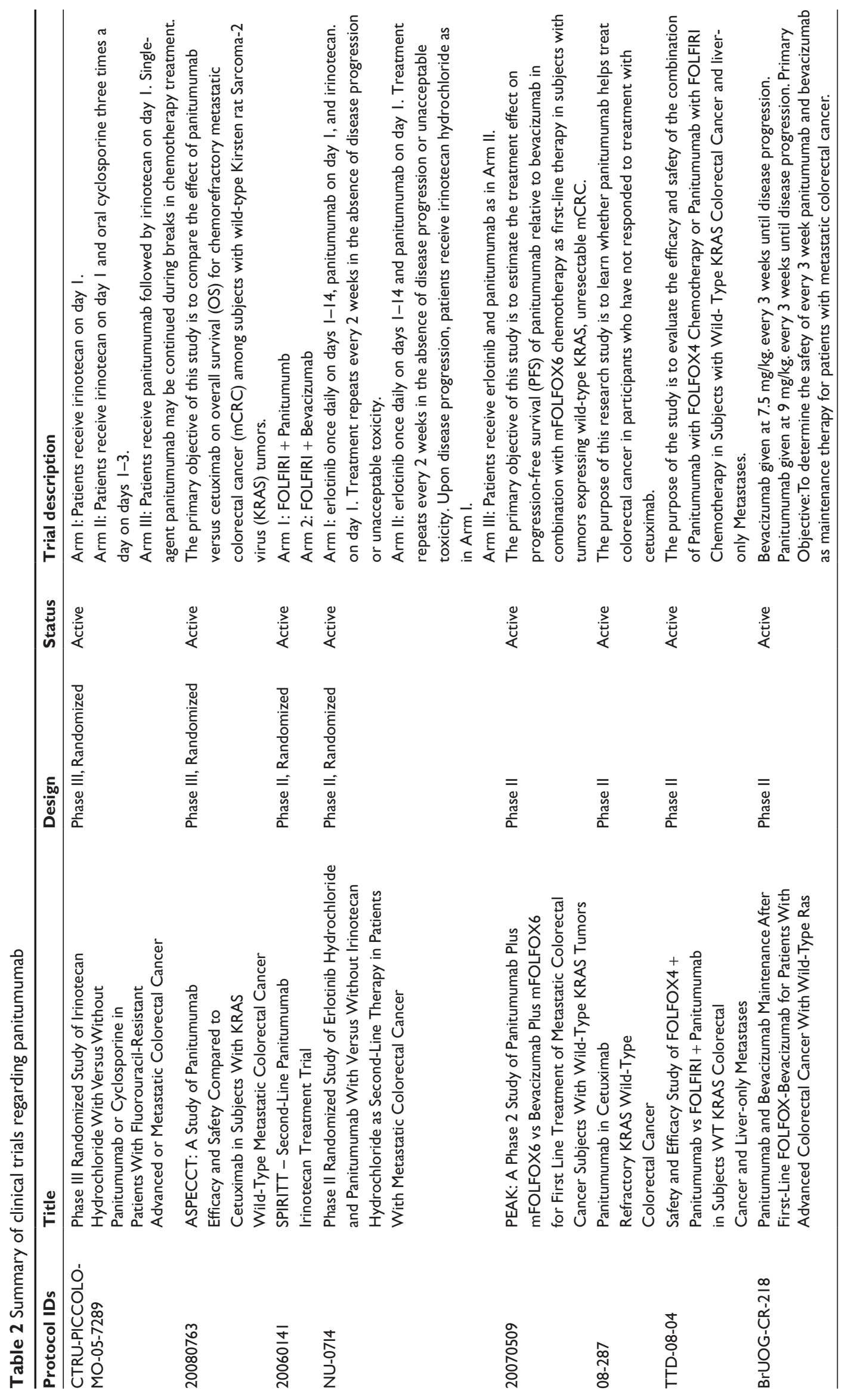


mutant. Wild-type K-RAS patients had a median PFS and response rate of 9.6 months and 55\% in Arm 1, and 8 months and $48 \%$ in Arm 2, respectively.

Patients with mutated K-RAS had a median PFS of 7.3 months in Arm 1 and 8.8 months in Arm 2. Moreover, response rate was improved in patients with Wild-type K-RAS tumors (55\% vs 48\%) and at interim analysis, OS seemed to be significantly improved in patients with Wild-type K-RAS tumors, although additional follow-up is required. Adverse events were similar across the two arms except for those that were associated with anti-EGFR therapy. Final results confirmed the importance of K-RAS as a predictive biomarker in the setting of first-line mCRC treatment with EGFR inhibitors. $^{21}$

The second study, performed by Peeters et al was a randomized, phase III study that evaluated the efficacy and safety of panitumumab with fluorouracil, leucovorin, and irinotecan (FOLFIRI) versus FOLFIRI alone as second-line treatment for mCRC. Patients enrolled in the study were randomized to receive panitumumab $6 \mathrm{mg} / \mathrm{kg}$ every 2 weeks plus FOLFIRI (Arm 1) versus FOLFIRI alone (Arm 2). Patients had metastatic colorectal adenocarcinoma; documented disease progression 6 months or less after 1 prior therapy with fluoropyrimidine for $\mathrm{mCRC}$, and ECOG score of $0-2$. The evaluation of PFS and OS by K-RAS mutational status were the primary endpoints in the study. A total of 1186 patients were randomized (Arm 1 = 591; Arm 2 = 595). Of all patients, 1803 (91\%) were evaluable for K-RAS, with 598 (55\%) being wild-type and 485 (45\%) mutated. PFS was longer in wild-type K-RAS patients who were in Arm 1 versus Arm 2 (5.9 vs 3.9 months), but was similar in K-RAS mutated patients (5.0 vs 4.9 months). A similar trend was seen with OS in wild-type and mutated patients when Arm 1 was compared to Arm 2 (wild-type, 14.5 vs 12.5 months; mutated, 11.8 vs 11.1 months). With regard to safety, panitumumab was well-tolerated with a manageable toxicity profile. ${ }^{22}$

\section{Ongoing clinical trials}

The study of panitumumab in CRC proceeds in a number of ongoing clinical trials. Current studies under way are evaluating panitumumab in combination with other chemotherapeutic drugs or with novel agents that have to come into common clinical practice. These trials will further define the role of panitumumab in CRC (Table 2). ${ }^{23}$

\section{Patient-oriented evidence EGFR expression}

Due to the mechanism of action of panitumumab, positive EGFR protein expression, as determined by immunohistochemistry (IHC), was initially selected as an entry criterion for several studies evaluating EGFR inhibitors. In this setting, data on the use of EGFR expression as a predictive biomarker of response to panitumumab therapy, showed controversial results. Two trials revealed a positive correlation between EGFR expression and response to panitumumab. In the first, Meropol et al ${ }^{24}$ enrolled 100 patients with metastatic colorectal cancer (mCRC) to evaluate panitumumab as monotherapy after failure of treatment with fluoropyrimidine plus irinotecan or oxaliplatin or both. Patients were eligible if $\geq 10 \%$ of the tumor cells had EGFR overexpression of $2+$ or $3+$ by IHC. $13 \%$ of patients had a PR, and their tumor cells had 3+ EGFR expression; 39\% of patients had a SD. Furthermore, panitumumab monotherapy was also evaluated in 300 patients with mCRC enrolled in a phase II trial after a disease progression despite treatment with fluoropyrimidine, irinotecan, and oxaliplatin-based chemotherapy. One of the inclusion criteria, was EGFR staining of $\geq 10 \%$ tumor cells on IHC. $8 \%$ of patients had PR, 21\% achieved SD, and disease progression was seen in $49 \%$ of patients. ${ }^{15}$

Controversial data were reported by Malik and Hecht in two separate trials. ${ }^{24,25}$

Malik et al conducted a phase II study enrolling two cohorts of patients with $\mathrm{mCRC}$ who failed chemotherapeutic regimens containing fluoropyrimidine plus irinotecan, oxaliplatin, or both. ${ }^{14}$ Cohort A consisted of patients with $2+$ or $3+$ EGFR over-expression in $\geq 10 \%$ of tumor cells and cohort $\mathrm{B}$ included patients with the sum of $2+$ and $3+$ in $<10 \%$ of tumor cells. PR was observed in $10 \%$ of patients (cohort A, 10\%; cohort B, 9\%). Overall median time to disease progression was 2.5 months $(95 \% \mathrm{CI}$, 2-4) (cohort A, 3.4 months (95\% CI, 2-4); cohort B, 2.1 months (95\% CI, 2-4.5). Overall median survival time was 9.4 months (95\% CI, 6.6-10.6) (cohort A, 10 months [95\% CI, 6.2-11]); cohort B, 9.4 months [95\% CI, 6-10.6]). Although no statistical analysis was performed to evaluate the differences between low and high EGFR-expressing tumors the response rates, time to disease progression, and survival time appeared similar irrespective of the level of EGFR expression.

Hecht et al conducted a phase II trial to assess response rates in patients with low ( $1 \%-9 \%$ of tumor cells) or negative ( $<1 \%$ of tumor cells) EGFR staining on IHC. ${ }^{25}$ The study enrolled patients with documented $\mathrm{mCRC}$ disease progression during or after 2 to 3 regimens of fluoropyrimidine, irinotecan, and oxaliplatin treatment. $7 \%$ of all patients had a PR (low EGFR expression, 8\%; negative EGFR expression, $6 \%$ ). $29 \%$ of all patients had SD (low EGFR expression, 
29\%; negative EGFR expression, 31\%), and disease control was observed in 37\% (low EGFR expression, 39\%; negative EGFR expression, 37\%). The median time to response was 9 weeks overall and in patients with low and negative EGFR expression, with a minimum of 7 weeks and a maximum of 12 weeks. Median duration of response was 20 weeks (range, 8-46 weeks) overall. The duration of response was longer in patients with negative EGFR expression: 31 weeks (range, 16-46 weeks) compared with 20 weeks (range, 8-20 weeks) in patients with low EGFR expression. Median PFS was 8 weeks overall (95\% CI, 7-11) (low EGFR expression, 8 weeks (95\% CI, 7-13); negative EGFR expression, 7 weeks (95\% CI, 7-14)).

Objective responses have been observed in patients with low or negative, as well as high, EGFR protein expression, thus suggesting that efficacy of panitumumab may not depend on the level of EGFR expression. Although immunohistochemistry is widely used, its usefulness in predicting the clinical benefit of panitumumab appears limited, probably because of several factors like the absence of a standardized scoring system and the variety on EGFR expression. Therefore, on this basis, the detection of positive EGFR expression actually does not reliably predict clinical outcome and requires further validation before incorporation into clinical practice.

\section{EGFR findings: affinity, phosphorylation, mutations and polymorphisms}

Several studies investigating other potential predictive biomarkers to EGFR inhibitors response are ongoing. Some of these, studied EGFR findings as low or high affinity or presence of phosphorylation to explain the variability of response to EGFR targeted therapy. In this setting, data exist for patients receiving cetuximab while actually we have no elements on panitumumab. Using a specific ligand binding assay, Francoual et $\mathrm{al}^{26}$ found that many tumors contain both low- and high-affinity EGFRs: $78 \%$ of 82 tumor specimens contained only high-affinity binding sites and $22 \%$ had both low- and high-affinity sites.

EGFR phosphorylation status may reflect the level of receptor utilization by the tumor and this parameter was associated with clinical response in patients treated with cetuximab-based therapy. Patients with an activated or phosphorylated EGFR score, as indicated by an immunohistochemistry-based visual score of 7 or greater, were almost twice as likely to have disease control (objective response or stable disease) than those with a score of less than $7(100 \%$ vs $54 \% ; P=0.05){ }^{27}$
EGFR mutations in mCRC account for less than $1 \%$ of tumors, therefore this measure is unlikely to be valid as a marker. ${ }^{28}$ Moreover, EGFR mutations that are associated with responses to tyrosine-kinase inhibitors in non-small cell lung cancer are not present in mCRC. ${ }^{29,30}$

Moroni et al detected one mutation (3.2\%) among 31 patients with $\mathrm{mCRC},{ }^{31}$ occurring in a patient who achieved SD for 24 weeks with cetuximab and chemotherapy treatment. This missense heterozygous mutation in exon 21 (Gly857Arg) affected a residue located within the activation loop of the EGFR catalytic domain and was one amino acid away from the Leu858Arg-activating mutation that has been identified in patients with lung cancer who respond to gefitinib or erlotinib. ${ }^{32}$ At disease progression, the patient whose tumor had this mutation was treated with gefitinib; this molecular alteration in EGFR was not associated with clinical response because the disease progressed after 4 weeks of treatment.

Notably, a specific polymorphism of EGFR affecting exon 13 at residue $521 \mathrm{Arg} / \mathrm{Arg}$ (previously identified as residue 497, rs11543848) has been linked with improved overall survival in women with metastatic colorectal cancer (vs Lys/Lys and/or Lys/Arg variants), although the reverse pattern was observed in men with this disease. ${ }^{33}$ This same polymorphism has been linked to cetuximab response in other studies ${ }^{34-36}$ while conflicting evidence also exists for a polymorphism affecting the ligand of EGFR, EGF, at position 61 (rs4444903). ${ }^{35-38}$ About panitumumab, data in this setting are related to recently analyses. Carcereny et al studied a cohort of $84 \mathrm{mCRC}$ patients receiving cetuximab or panitumumab. A single nucleotide polymorphism (SNP) at codon 497 (497 G/A) was associated with worse RR, PFS and OS, and therefore could be a resistance factor. ${ }^{39}$ More recently a retrospectively analyses has not revealed the same conclusion of Carcereny et al. In a total of 117 patients treated with cetuximab or panitumumab, there were no significant differences on response rate $(9 / 59 ; 15.2$ vs $9 / 52 ; 17.3 \%)$, PFS (13.5 vs $13.2 \mathrm{w}$ ) and OS (33 vs $26.8 \mathrm{w}$ ) according to EGFR R497K (GG vs GA/AA). Despite, the predictive role of K-RAS mutational status was confirmed. ${ }^{40}$ On this basis, actually we have no certain data about these EGFR findings, so further investigations need to better define their exact role in EGFR inhibitors response.

\section{EGFR amplification}

A small proportion of colorectal tumors over-express EGFR via amplification of the gene, which can be detected by fluorescence in situ hybridization (FISH) or chromogenic in situ hybridization. ${ }^{41}$ Available data suggest that patients with less 
than three EGFR gene copies per nucleus have a relatively low likelihood of responding to EGFR-targeted monoclonal antibody treatment. ${ }^{42-47}$

Despite when EGFR gene copy number was evaluated by polymerase chain reaction, no association was found between this parameter and clinical outcome of panitumumab- or cetuximab-based treatment, ${ }^{48,49}$ probably because of tumor DNA dilution by DNA from normal cells during DNA extraction. However, EGFR gene copy number as analyzed by FISH or chromogenic in situ hybridization appears to be a promising biomarker of response to such treatment. In a retrospective analysis of a subgroup of patients participating in the pivotal phase III trial of panitumumab monotherapy, ${ }^{50}$ the mean EGFR gene copy number per nucleus and the percentage of tumor cells with chromosome 7 polysomy (three or more EGFR signals per nucleus) were analyzed by FISH and the association between these parameters and clinical outcome was assessed. None of the patients with a mean of $<2.47$ EGFR gene copies per nucleus or fewer than $43 \%$ of tumor cells with chromosome 7 polysomy, respectively, achieved objective response compared with $30 \%$ of the patients $(P=0.001)$ and $32 \%$ of the patients $(P=0.001)$ who had values above these thresholds.

A mean EGFR gene copy number threshold of less than 2.5 copies per nucleus or fewer than $40 \%$ of tumor cells with chromosome 7 polysomy discriminated patients with shorter progression-free $(P=0.039$ and $P=0.029$, respectively) and overall survival $(P=0.015$ and $P=0.014$, respectively). EGFR gene copy number and chromosome 7 polysomy status did not draw a parallel with progressionfree interval in patients receiving only supportive care in this study, suggesting that this parameter is not prognostic in metastatic colorectal cancer. Homogeneous (ie, 100\%) chromosome 7 disomy was the most common pattern found in 58 colorectal tumors with non increased gene copy number $(\mathrm{n}=26 ; 45 \%)$. Chromosome 7 disomy is also easier to detect than an increase in EGFR gene copy number and therefore, might enable a more reproducible FISH assay. For instance, Moroni et $\mathrm{al}^{31}$ found a $89 \%$ response rate in a subgroup of nine patients with colorectal cancer whose tumors had an increased EGFR gene copy number, but these investigators included a relatively high proportion of responders (9 of 29 patients; 31\%) in their analysis.

In comparison with patients with normal EGFR gene copy number, patients with an increased EGFR gene copy number exhibit higher response rates to EGFR-targeted monoclonal antibodies, with a longer progression-free interval or time to progression. These results have to be confirmed by further analyses before the incorporation of this promising parameter into clinical practice.

\section{EGFR ligands}

Several preclinical studies have found that cetuximab decreases levels of epiregulin (ER) and amphiregulin (AR) that are two EGFR ligands even more powerful than EGF for activating EGFR. Therefore the possible predictive roles as biomarkers to selecting patients have been investigated in trials involving cetuximab. ${ }^{49-52}$ All data of these studies suggest that cetuximab treated patients with high ER and AR expression levels obtained a better response rate and PFS. Recently the association of a high epiregulin gene expression with a K-RAS status wild-type seems to be more predictive of cetuximab benefit in the treatment of mCRC than these markers analyzed alone. Further studies are needed but the authors suggest that determination of epiregulin gene expression levels should be prospectively evaluated in patient selection for EGFR targeted therapy. ${ }^{53}$

\section{K-RAS mutations}

The K-RAS protein, encoded by K-RAS, is a GTPase that regulates different signaling pathways. This protein may be active (RAS-GTP) or inactive (RAS-GDP). K-RAS mutations yield a defective GTPase activity and then an increased population of active K-RAS protein, activating signaling in two pathways, PI3K/PTEN/AKT and RAF/MEK/ERK which are involved in cell proliferation, survival and angiogenesis. K-RAS is mutated in approximately $30 \%-50 \%$ of colorectal cancer; the most common and clinically relevant K-RAS mutations are nonsense somatic alterations which have been described at codons 12 (about $82 \%$ of cases) and 13 (about 13\% of cases) in exons 2 of the K-RAS gene; additional mutations can be found at codon 61 . All these mutations are associated with cancer progression. Recent studies have revealed that mutation status of K-RAS has emerged as both an important predictor of response to EGFR inhibitors, including panitumumab, and a marker for patient selection. ${ }^{54}$

Benvenuti et al analyzed tumor K-RAS status from 48 patients with $\mathrm{mCRC}$ treated with panitumumab or cetuximab. ${ }^{55}$ Presence of K-RAS mutations (exon 2) were detected in $33,3 \%$ of tumors and it was not significantly linked to objective response to therapy, with a trend toward a negative association with response (1 of 11 mutations versus 15 of 37 mutations for responders versus non-responders; $P=0.073$ ); consequently time to progression analysis showed a significantly worse outcome for subjects bearing a mutated $\mathrm{K}-\mathrm{RAS}$ allele in their tumors compared with those carrying 
wild-type K-RAS $(P=0.0443)$. In this study the authors also found that the transfection of mutated K-RAS (G12V) into wild-type cellular models of colorectal cancer confers resistance to the treatment with cetuximab.

A confirm of K-RAS as a predictive marker to therapy with panitumumab was reported by Amado et $\mathrm{al}^{56}$ in a randomized phase III trial setting. Among the 463 patients enrolled in this study, 427 (92\%) were included in the K-RAS analysis. Of these 427, 184 (43\%) were found to have tumors harboring mutant K-RAS. Among the 208 patients assigned to panitumumab, $17 \%$ of the 124 patients in the wild-type K-RAS subgroup achieved objective response, whereas none of the 84 patients in the mutant K-RAS subgroup responded to this treatment. Median progression-free interval among those treated with panitumumab was 12.3 weeks among those in the wild-type K-RAS subgroup and 7.4 weeks among those in the mutant K-RAS subgroup. The OS time was also longer in patients with wild-type K-RAS tumors, 8.1 months, versus 4.9 months. The hazard ratio (HR) for disease progression or death (panitumumab vs control group) was $0.45(95 \% \mathrm{CI}=0.34$ to 0.59$)$ for panitumumab in the wild-type K-RAS subgroup, but there was no benefit of panitumumab in the mutant K-RAS subgroup ( $\mathrm{HR}=0.99$, $95 \% \mathrm{CI}=0.73$ to 1.36$)$.

Similar findings in term of predictive role of K-RAS status to therapy with panitumumab were observed by Hecht et al. ${ }^{16}$ In this study, 171 patient samples were available for K-RAS tumor presence. The overall response rate was significantly better in patients with wild-type K-RAS tumors (9\% vs 0\%). Median PFS was significantly longer in patients with wild-type K-RAS tumors than in patients with mutant K-RAS tumors (15 weeks vs 7.1 weeks, respectively). This study also demonstrated a significant benefit in terms of OS in patients with wild-type K-RAS tumors treated with panitumumab when compared with patients with mutant K-RAS tumors (13.5 months vs 7.3 months, respectively).

Freeman et al retrospectively analyzed 62 patients from three phase II studies with panitumumab in mCRC patients. ${ }^{57}$ K-RAS mutation was found in $38.7 \%$ of them. In the wild-type K-RAS group, $11 \%$ of patients had a PR, 53\% had SD, and $37 \%$ had progressive disease. In the mutant K-RAS group, $21 \%$ of patients had SD and $79 \%$ of patients had PD; there were no responses. The absence of a K-RAS mutation was associated with response to panitumumab (PR vs SD vs progressive disease; $P=0.0028$ ). The HR for wild-type versus mutant K-RAS was 0.4 (95\% CI, 0.2-0.7) for progression-free survival (PFS) and 0.5 (95\% CI, 0.3-0.9) for OS. Second-line treatment with panitumumab and FOLFIRI by tumor K-RAS status in patients with mCRC was also investigated; in interim analyses, numerical significantly differences in PFS (26 vs 16 weeks) and median OS (39 vs 31 weeks) in favour of patients with wild-type K-RAS were observed. ${ }^{58}$

More recently the efficacy of panitumumab by tumor K-RAS status was investigated in some phase III trials. Douillard et al enrolled 1183 patients to evaluate the efficacy of FOLFOX-4 with or without panitumumab as first line treatment in patients with mCRC (PRIME trial). ${ }^{21}$ Results showed that the addition of panitumumab to chemotherapy improved RR (55\% vs 48\%) and PFS (9.6 vs 8.0 months; HR = 0.80; 95\% CI: $0.66-0.97 ; P=0.02$ ) in patients with wild-type K-RAS. No benefit in RR and PFS from the addition of panitumumab was noticed in patients with K-RAS mutations. Interim OS showed an improvement only for patients with wild-type K-RAS tumors $(\mathrm{HR}=0.83, P=0.16)$.

Similar results were reported in 1186 patients enrolled in a randomized phase III study which had the aim to evaluate the association of panitumumab with FOLFIRI as second-line treatment in patients with $\mathrm{mCRC} .{ }^{22}$ In patients with wild-type K-RAS tumors, panitumumab significantly improved response rate (35\% vs 10\%) and PFS (median 5.9 vs $3.9 \mathrm{mo}$; $\mathrm{HR}=0.73, \mathrm{p}=0.004$ ) when added to FOLFIRI. OS was also improved in patients with wild-type K-RAS tumors with panitumumab plus FOLFIRI (median 14.5 vs $12.5 \mathrm{mo} ; \mathrm{HR}=0.85, P=0.12$ ).

There was no evidence of benefit in patients with mutated K-RAS tumors. In a recently randomised phase III trial which evaluated bevacizumab and chemotherapy with or without panitumumab in $\mathrm{mCRC},{ }^{20}$ the predictive role of K-RAS status was confirmed; and progression-free interval was worse among patients with tumors carrying wild-type K-RAS (11.5 months vs 9.8 months in the panitumumab arm).

A recent exploratory analysis investigating the combination of panitumumab with FOLFIRI as first line-treatment in K-RAS wild-type patients, appears to show improvement in PFS and time to disease progression versus the K-RAS mutation population. ${ }^{59}$

As far as cetuximab, across all studies reviewed here, it clearly appears that clinical efficacy of panitumumab therapy is restricted to patients with wild-type K-RAS tumors. Therefore, K-RAS genotyping of tumors should be strongly considered to select patients being treated with panitumumab.

Further evaluations need to assess the relationship between K-RAS mutations and response to panitumumab combined with chemotherapy in earlier lines of therapy. 


\section{B-RAF}

The B-Raf proto-oncogene serine/threonine-protein kinase (B-RAF) is involved in transducing mitogenic signals via the MAP kinase/ERK (MAPK) signaling pathway. Mutations in B-RAF are involved in mCRC. A thymine to adenine transversion mutation results in the substitution of valine with glutamate (V600E) and converts B-RAF into a dominant transforming protein that causes the constitutive activation of the MAPK pathway independently of RAS. The V600E B-RAF mutation appears in $4 \%-15 \%$ of CRC. ${ }^{60-62}$

The possible relationship between of B-RAF mutational status and response to treatment with panitumumab in 48 patients with $\mathrm{mCRC}$ was investigated by Benvenuti et al. ${ }^{55}$ B-RAF mutations were detected in $12,5 \%$ tumors and they were mutually exclusive with K-RAS alterations. The only B-RAF mutation found was the V600E substitution. The most important thing was that patients who received panitumumab or cetuximab but had B-RAF alteration, presented no objective response to therapy; consequently TTP was worse for subjects bearing a mutated B-RAF although not statistically significant probably due to the limited number of tumors carrying these mutations.

More recently Di Nicolantonio et al analysed response rate, PFS, OS and the mutational status of K-RAS and B-RAF in 113 tumors from cetuximab- or panitumumab-treated mCRC patients. ${ }^{63} \mathrm{~K}-\mathrm{RAS}$ was mutated in $30 \%$ of cases and B-RAF was mutated in $14 \%$ of the K-RAS-wt cases. None of the B-RAF mutated patients responded to treatment, and none of the responders carried any B-RAF mutations. B-RAF mutated patients had a significantly shorter PFS and OS than the wild-type cases. Di Nicolantonio et al also demonstrated that introduction of the B-RAF V600E allele could confer resistance to either cetuximab or panitumumab in wild-type B-RAF colorectal cancer cells.

Thus, the low incidence of B-RAF mutations in patients with $\mathrm{mCRC}$ probably do not permit to select patients who have been treated with panitumumab. In clinical practice, a B-RAF alteration could explain the resistance to anti-EGFR inhibitors in a $10 \%$ subpopulation who have K-RAS wildtype; however, $41 \%$ of patients had no mutations in either K-RAS or B-RAF and did not respond to therapy, therefore other biomarkers are required.

\section{Alternative K-RAS pathways: PI3K, PTEN/AKT}

The PIK3CA gene encodes for a lipid kinase that regulates, alongside with K-RAS, signaling pathways downstream of the EGFR. The PIK3CA gene is mutated in $10 \%-18 \%$ of mCRC cases. ${ }^{64,65} \mathrm{PI} 3 \mathrm{~K}$-initiated signaling is normally inhibited by phosphatase and tensin homolog deleted on chromosome ten (PTEN). Thus, PTEN functions as a tumor suppressor gene through the action of its phosphatase protein product. The encoded protein negatively regulates intracellular levels of phosphatidylinositol-3,4,5-trisphosphate (PIP-3) in cells and functions as a tumor suppressor by negatively regulating the PI3K/AKT signaling pathway. PTEN loss increases levels of PIP-3 and PKB/AKT, thus increasing cell survival signalling. ${ }^{66,67}$

Several studies have evaluated the possible role of PI3K and PTEN as predictive biomarkers of anti-EGFR drug activity, especially in patients receiving cetuximab, but data seem more controversial. ${ }^{68-71}$ In $\mathrm{mCRC}$, it has been reported that loss of PTEN expression, which occurs in 30\% of sporadic cases, may be associated with lack of response to cetuximab.

Our present knowledge of the active pathways for panitumumab is based on a single study conducted by SartoreBianchi. ${ }^{72}$ In this analysis, mutational profiling of $110 \mathrm{CRC}$ tumors from patients receiving cetuximab or panitumumab led to the identification of $13.6 \%$ PIK3CA and $29.0 \% \mathrm{~K}$-RAS mutations. The study showed that mutations in PIK3CA, $\mathrm{K}-\mathrm{RAS}$, and PTEN loss were associated with lack of objective response to panitumumab or cetuximab. PIK3CA mutations were significantly associated with lack of response to panitumumab or cetuximab, with none of the mutated patients achieving objective tumor response $(P=0.038)$.

The same negative association was confirmed for K-RAS mutations $(9.1 \%$ of mutations among responders versus $34.5 \%$ among non responders; $P=0.019$ ) and was confirmed when at least a mutation of either K-RAS or PIK3CA was considered $(P=0.001)$. Consequently patients with tumors harboring PIK3CA mutations had a worse clinical outcome in terms of PFS, compared with wild-type tumors $(P=0.0035)$. Patients with K-RAS mutations had a trend toward a decreased PFS $(P=0.0815)$. Shorter PFS was also detected in patients harboring at least a mutation of either K-RAS or PIK3CA $(P=0.0032)$.

Despite the results of this study, further investigation is needed to establish the effective roles of PI3K and PTEN/ AKT as predictive biomarkers to selecting patients who could have be treated with EGFR inhibitors, including panitumumab.

\section{Other potential biomarkers}

Increased gene copy number of HER2 (the preferred heterodimer of EGFR) was linked to a statistically significantly 
shorter overall survival $(P=0.03)$, with a trend toward a shorter time to progression $(P=0.09)$, in 85 patients receiving cetuximab with or without chemotherapy. ${ }^{73}$

The interaction between IGF-1 expression and K-RAS mutational analysis was also tested in order to verify the ability of IGF-1 to identify a sub-group of patients more likely to benefit from EGFR-targeted antibodies treatment. ${ }^{74}$ Among K-RAS wild type patients, median time to progression in IGF-1 negative tumors was 11 months and 3.2 months in IGF-1 positive CRC $(P$ $=0.03$ ). IGF-1 proved to be a reliable predictive factor for resistance to anti-EGFR monoclonal antibodies in K-RAS wild type CRC. Thus combined IGF-1 and K-RAS analysis may represent an effective strategy for a better selection of responding CRCs in this setting. Other potential mechanisms of acquired resistance to EGFR inhibitors, such as those involving activation HER3, mesenchymal-epithelial transition factor (C-MET), MAPK, AKT, VEGF, IL8, COX-2 and Cyclin D are under investigation in patients with $\mathrm{mCRC}$ receiving EGFR inhibitors but data on their use in selecting patients are not available. ${ }^{75-77}$

\section{Skin toxicity}

Skin toxicity is most frequently seen as acneiform rash generally confined to the seborrhoeic areas; this particularly toxicity is a characteristic finding seen with most EGFR inhibitors, including panitumumab. It appears to be dose-related and may indicate EGFR saturation, given the high expression of this receptor in keratinocytes skin fibroblasts and hair follicles. Skin rash observed in most patients who have been treated with EGFR inhibitors has been studied as a potential marker of efficacy. Skin toxicity has been significantly linked with higher response rate and longer survival in several trials with patients with $\mathrm{mCRC}$ and treated with cetuximab.

Longer PFS and OS were significantly observed in patients with worst skin toxicity of grade 2-4 compared with those with at worst grade $1 .{ }^{78,79}$ The association between skin toxicity and PFS was only seen for panitumumab patients with wild-type K-RAS. No association between skin toxicity and PFS was seen in the mutant K-RAS group. Of note, a higher incidence of grade 3 skin toxicity was observed in patients with wild-type K-RAS tumors as compared with mutant K-RAS, consistent with longer time on treatment. The association of OS with skin toxicity severity was more pronounced for the wild-type K-RAS than the mutant K-RAS group.

Berlin et al conducted a pooled analysis of five clinical trials which involved a total of 612 patients with $\mathrm{mCRC}$ treated with panitumumab. ${ }^{80}$ They showed better overall response rate, $\mathrm{PFS}$, and OS in patients who developed a grade 2-4 skin toxicity than in patients with grade $0-1$ skin toxicity.
Although there is some evidence that skin toxicity represents a marker of clinical benefit in patients treated with panitumumab, its utility in the clinical setting is limited, as it cannot be used a priori to select patients who may derive greater benefit from anti-EGFR treatment, or conversely, exclude those who may not. Potentially, however, this marker could be used clinically to titrate treatment doses to achieve a skin toxicity grade consistent with maximum treatment benefit.

\section{Economic evidence}

Because of substantial increase in costs, physicians need to consider the cost-effectiveness of new therapies as well as the clinical issues. Consideration of a treatment's cost effectiveness can help to avoid therapies that produce too little benefit at too high a cost.

More progress has been made in increasing the duration of survival in patients with $\mathrm{mCRC}$ in the past 5 years than in most other cancers. Although the introduction of better systemic therapy and novel therapeutic agents has considerably improved the prognosis in this setting, these potential clinical benefits caused escalating drug costs. The near-doubling of the median survival time achieved over the past decade has been accompanied by a 340 -fold increase in drug costs for the initial 8 weeks of therapy alone. ${ }^{81}$ Many countries are experiencing increased pressure on their budgets to finance these therapies.

Conversely, Paramore et al showed that the economic impact of mCRC is substantial and increasing over time, and that monthly cost almost tripled from 1998 to $2004 .^{82}$ Largescale economic analyses reported that employing cetuximab in patients with metastatic colorectal cancer is clinically effective but associated with high costs per life year.

Regarding panitumumab, the average wholesale price for panitumumab $(20 \mathrm{mg} / \mathrm{mL} 20 \mathrm{~mL}$ vial $)$ is US $\$ 4000$. Therefore, the cost of therapy for a $60 \mathrm{~kg}$ patient is about $\$ 8000 /$ month.

A recent Italian treatment costs analysis to evaluate the safety of panitumumab comparing with cetuximab in third line mCRC, identified savings per patient of 111 euro per month. ${ }^{83}$ The safety savings were 50 euro per month and the administration savings were 690 euro per month, giving a combined saving of 851 euro. Treatment cost savings associated with panitumumab were related to a low rate of severe infusion reaction versus cetuximab, reduced hospital costs associated with a less frequent dosing regimen and patients' weight savings across a normally distributed patient population. Based on the calculated prevalence and the assumption of a 4 month treatment period, panitumumab is modeled to provide savings of 7.67 million euro/year to the Italian healthcare system compared 
with cetuximab. Reduced drug costs and further improvements in clinical effectiveness may alter this finding. ${ }^{84,85}$

Moreover, K-RAS mutational status has emerged as an important biomarker for $\mathrm{mCRC}$ and should be assessed before patients begin therapy. ${ }^{86}$ Because K-RAS status can aid in therapy selection, oncologists can avoid unnecessary toxicities and expenses related to patients unlikely to respond. A recent study by Mancl et al reviewed the clinical use of cetuximab and panitumumab and the role of K-RAS testing in clinical practice. ${ }^{87} \mathrm{~A}$ simple breakeven analysis using a group of 100 hypothetical patients with metastatic colorectal cancer revealed that Preemptive K-RAS screening had tremendous cost-saving potential.

Screening could cost several thousand dollars per patient and still result in a lower overall cost of care, based on very conservative estimates of the cost reduction associated with treatment avoidance in patients with K-RAS mutations. Moreover, because EGFR inhibitors are indicated as thirdline therapy for metastatic colorectal cancer, few options exist for patients who do not respond to treatment or have mutant K-RAS. Such patients will most likely receive best supportive care or choose to enroll in a clinical trial. ${ }^{87}$

Implementing routine K-RAS screening and limiting the use of EGFR inhibitors to patients with wild-type (not mutated) K-RAS may have the potential for cost savings.

\section{Conclusions}

The evidence to hand and the implications of these are summarized from the above discussion into clinical impact summary.

Intravenous panitumumab has been approved as monotherapy for the treatment of adult patients with chemotherapy-refractory, EGFR-expressing, mCRC with non mutated K-RAS.

The improvement in PFS and RR produced by panitumumab monotherapy was significantly greater in patients with non mutated (wild-type) K-RAS than in those with mutant K-RAS in whom no benefit from panitumumab was observed.

The predictive value of mutant K-RAS for a lack of clinical benefit with panitumumab monotherapy was supported and also confirmed by results recently from some phase II-III studies. Therefore K-RAS status evaluation is the only sure predictive biomarker of response that have to be investigated before starting a therapy with EGFRinhibitors in patients with mCRC. Thus implementing routine K-RAS screening and limiting the use of EGFR inhibitors to patients with wild-type K-RAS appears the better strategy for selecting only patients who could benefit from the therapy with panitumumab and also may have the potential for significant cost savings while improving positive outcomes for patients.

Uncertain data are about new biomarkers, such as B-RAF or PTEN, which may play a role in predictive response, that will further narrow the selection of $\mathrm{mCRC}$ patients.

\section{Disclosure}

The authors report no conflicts of interest in this work.

\section{References}

1. Overman MJ, Hoff PM. EGFR-targeted therapies in colorectal cancer. Dis Colon Rectum. 2007;50:1259-1270.

2. Mayer A, Takimoto M, Fritz E, et al. The prognostic significance of proliferating cell nuclear antigen, epidermal growth factor receptor, and $m d r$ gene expression in colorectal cancer. Cancer. 1993;71:2454-2460.

3. Giusti RM, Shastri KA, Cohen MH, et al. FDA drug approval summary: panitumumab (Vectibix). Oncologist. 2007;12:577-583.

4. Hoy SM, Wagstaff AJ. Panitumumab: in the treatment of metastatic colorectal cancer. Drugs. 2006;66:2005-2014.

5. Baselga J, Arteaga CL. Critical update and emerging trends in epidermal growth factor receptor targeting in cancer. J Clin Oncol. 2005;23:2445-2459.

6. Yang XD, Jia XC, Corvalan JR, et al. Development of ABX-EGF, a fully human anti-EGF receptor monoclonal antibody, for cancer therapy. Crit Rev Oncol Hematol. 2001;38:17923.

7. Foon KA, Yang XD, Weiner LM, et al. Preclinical and clinical evaluations of ABX-EGF, a fully human anti-epidermal growth factor receptor antibody. Int J Radiat Oncol Biol Phys. 2004;58:984-990.

8. Ranson M. Technology evaluation: ABX-EGF, Abgenix/Amgen. Curr Opin Mol Ther. 2003;5:541-546.

9. Carteni G, Fiorentino R, Vecchione L, et al. Panitumumab a novel drug in cancer treatment. Ann Oncol. 2007;18 Suppl 6:vi16-vi21.

10. Pfeiffer P, Qvortrup C, Eriksen JG. Current role of antibody therapy in patients with metastatic colorectal cancer. Oncogene. 2007;26: 3661-3678.

11. Rowinsky EK, Schwartz GH, Gollob JA, et al. Safety, pharmacokinetics, and activity of ABX-EGF, a fully human anti-epidermal growth factor receptor monoclonal antibody in patients with metastatic renal cell cancer. J Clin Oncol. 2004;22:3003-3015.

12. Figlin RA, Belldegrun AS, Crawford J, et al. ABX-EGF, a fully human anti-epidermal growth factor receptor monoclonal antibody in patients with advanced cancer: phase 1 clinical results. Proc Am Soc Clin Oncol. 2002;Abstr 35.

13. Weiner L, Belldegrun A, Rowinsky E, et al. Update results from a dose and schedule study of panitumumab (ABX-EGF) monotherapy, in patients with advanced solid malignancies. J Clin Oncol. 2005;23:16S (abstract 3059).

14. Malik I, Hecht JR, Patnaik A, et al. Safety and efficacy of panitumumab monotherapy in patients with metastatic colorectal cancer (mCRC) (abstract). Proc ASCO. 2005;23:3520.

15. Berlin J, Neubauer M, Swanson P, et al. Panitumumab antitumor activity in patients (pts) with metastatic colorectal cancer (mCRC) expressing $\geq 10 \%$ epidermal growth factor receptor (EGFr) (abstract). Proc ASCO. 2006;24:3548.

16. Hecht J, Mitchell E, Baranda J, Richards D, et al. Panitumumab (pmab) efficacy in patients (pts) with metastatic colorectal cancer (mCRC) with low or undetectable levels of epidermal growth factor receptor (EGFr): final efficacy and KRAS analyses. ASCO Gastrointestinal Cancers Symposium. Orlando, USA: 2008.

17. Hecht J, Posey J, Tchekmedyian S, et al. Panitumumab in combination with 5-fluorouracil, leucovorin, and irinotecan (IFL) or FOLFIRI for firstline treatment of metastatic colorectal cancer (mCRC). ASCO Gastrointestinal Cancers Symposium. San Francisco, USA: 2006. Abstr. 237. 
18. Van Cutsem E, Peeters M, Siena S, et al. Open-label phase III trial of panitumumab plus best supportive care compared withbest supportive care alone in patients with chemotherapy refractory metastatic colorectal cancer. J Clin Oncol. 2007;25:1658-1664.

19. Van Cutsem E, Siena S, Humblet Y, et al. An open-label single arm study assessing safety and efficacy of panitumumab in patients with metastatic colorectal cancer refractory to standard chemotherapy. Ann Oncol. 2008;19:92-98.

20. Hecht JR, Mitchell E, Chidiac T, et al. A randomized phase III of chemotherapy, bevacizumab, and panitumumab a compared with chemotherapy and bevacizumab alone for metastatic colorectal cancer. J Clin Oncol. 2009;27:672-680.

21. Douillard J, Siena S, Cassidy J, et al. Randomized phase III study of panitumumab with FOLFOX4 compared to FOLFOX4 alone as 1st-line treatment (tx) for metastatic colorectal cancer (mCRC): the PRIME trial Joint ECCO/ESMO Multidisciplinary Congress. Berlin: 2009; Abstr. 10-LBA.

22. Peeters M, Price T, Hotko Y, et al. Randomized phase III study of panitumumab with FOLFIRI vs FOLFIRI alone as second-line treatment (tx) in patients (pts) with metastatic colorectal cancer (mCRC). Joint ECCO/ESMO Multidisciplinary Congress. Berlin: 2009. Abstr. 14-LBA.

23. http://www.cancer.gov/clinicaltrials/search

24. Meropol NJ, Berlin J, Hecht JR, et al. Multicenter study of ABX-EGF monotherapy in patients with metastatic colorectal cancer. Proc ASCO. 2003;Abstr. 1026.

25. Hecht JR, Patnaik A, Malik I, et al. ABX-EGF monotherapy in patients (pts) with metastatic colorectal cancer (mCRC): An updated analysis. Proc ASCO. 2004;Abstr. 3511

26. Francoual M, Etienne-Grimaldi MC, Formento JL, et al. EGFR in colorectal cancer: more than a simple receptor. Ann Oncol. 2006;17(6): 962-967

27. Personeni N, Hendlisz A, Gallez J, et al. Correlation between the response to cetuximab alone or in combination with irinotecan and the activated/phosphorylated epidermal growth factor receptor in metastatic colorectal cancer. Semin Oncol. 2005;32(6):S59-S62.

28. Khambata-Ford S, Garret C, Meropol N, et al. Expression of epiregulin and amphiregulin and KRAS mutation status predict disease control in metastatic colorectal cancer patients treated with cetuximab. J Clin Oncol. 2007;25:3230-3237.

29. Janne PA, Engelman JA, Johnson BE. Epidermal growth factor receptor mutations in nonsmall-cell lung cancer: implications for treatment and tumor biology. J Clin Oncol. 2005;23:3227-3234.

30. Tsuchihashi Z, Khambata-Frd S, Hanna N, et al. Responsiveness to cetuximab without mutations in EGFR. $N$ Engl J Med. 2005;353: 208-209.

31. Moroni M, Veronese S, Benevenuti S, et al. Gene copy number for epidermal growth factor receptor (EGFR) and clinical response to anti-EGFR treatment in colorectal cancer: a cohort study. Lancet Oncol. 2005;6:279-286.

32. Moroni M, Sartore-Bianchi A, Benvenuti S, et al. Somatic mutation of EGFR catalytic domain and treatment with gefitinib in colorectal cancer. Ann Oncol. 2005;16(11):1848-1849.

33. Press OA, Zhang W, Gordon MA, et al. Gender-related survival differences associated with EGFR polymorphisms in metastatic colon cancer. Cancer Res. 2008;68(8):3037-3042.

34. Goncalves A, Esteyries S, Taylor-Smedra B, et al. A polymorphism of EGFR extracellular domain is associated with progression free-survival in metastatic colorectal cancer patients receiving cetuximab-based treatment. BMC Cancer. 2008;8:1-11

35. Graziano F, Ruzzo A, Loupakis F, et al. Pharmacogenetic profiling for cetuximab plus irinotecan therapy in patients with refractory advanced colorectal cancer. J Clin Oncol. 2008;26(9):1427-1434.

36. Wang WS, Chen PM, Chiou TJ, et al. Epidermal growth factor receptor $\mathrm{R} 497 \mathrm{~K}$ polymorphism is a favorable prognostic factor for patients with colorectal carcinoma. Clin Cancer Res. 2007;13(12): 3597-3604.
37. Garm Spindler KL, Pallisgaard N, Rasmussen AA, et al. The importance of KRAS mutations and EGF61A > G polymorphism to the effect of cetuximab and irinotecan in metastatic colorectal cancer. Ann Oncol. 2009;20(5):879-884.

38. Zhang W, Gordon M, Press OA, et al. Cyclin D1 and epidermal growth factor polymorphisms associated with survival in patients with advanced colorectal cancer treated with cetuximab. Pharmacogenet Genomics. 2006;16(7):475-483.

39. Carcereny E, Castellvi-Bel S, Alonso V, et al. EGFR polymorphisms as predictors of clinical outcome in patients with advanced colorectal cancer treated with cetuximab and panitumumab. J Clin Oncol. 2008;26s:Abstr. 4124.

40. Maurel J, Alonso-Espinaco V, Alonso V, et al. EGFR polymorphism and KRAS mutational status as predictors of resistance to anti-EGFR therapy in advanced colorectal cancer (ACRC): A GEMCAD study. J Clin Oncol. 2009;26:Abstr. 4060.

41. Shia J, Klimstra DS, Li AR, et al. Epidermal growth factor receptor expression and gene amplification in colorectal carcinoma: an immunohistochemical and chromogenic in situ hybridization study. Mod Pathol. 2005;18(10):1350-1356.

42. Lievre A, Bachet JB, Le Corre D, et al. K-RAS mutation status is predictive of response to cetuximab therapy in colorectal cancer. Cancer Res. 2006;66(8):3992-3995.

43. Personeni N, Fieuws S, Piessevaux H, et al. Clinical usefulness of EGFR gene copy number as a predictive marker in colorectal cancer patients treated with cetuximab: a fluorescent in situ hybridization study. Clin Cancer Res. 2008;14(18):5869-5876.

44. Capuzzo F, Finocchiaro G, Rossi E, et al. EGFR FISH assay predicts for response to cetuximab in chemotherapy refractory colorectal cancer patients. Ann Oncol. 2008;19:717-723.

45. Capuzzo F, Varela-Garcia M, Finocchiaro G, et al. Primary resistance to cetuximab therapy in EGFR FISH-positive colorectal cancer patients. Br J Cancer. 2008;99:83-89.

46. Goncalvez A, Esteyries S, Taylor-Smedra B, et al. A polymorphism of EGFR domain is associated with progression free-survival in metastatic colorectal cancer patients receiving cetuximab based treatment. BMJ Cancer. 2008;8:169.

47. Frattini M, Saletti P, Romagnani E, et al. PTEN loss of expression predicts cetuximab efficacy in metastatic colorectal cancer patients. Br J Cancer. 2007;97:1139-1145.

48. Lenz HJ, Van Cutsem E, Khambata-Ford S, et al. Multicenter phase II and translational study of cetuximab in metastatic colorectal carcinoma refractory to irinotecan, oxaliplatin and fluoropyrimidines. J Clin Oncol. 2006;24:4914-4921.

49. Khambata-Ford S, Garret C, Meropol N, et al. Expression of epiregulin and amphiregulin and KRAS mutation status predict disease control in metastatic colorectal cancer patients treated with cetuximab. J Clin Oncol. 2007;25:3230-3237.

50. Sartore-Bianchi A, Moroni M, Veronese S, et al. Epidermal growth factor receptor gene copy number and clinical outcome of metastatic colorectal cancer treated with panitumumab. J Clin Oncol. 2007; 25(22):3238-3245.

51. Tejpar S, De Roock W, Biesmans B, et al. High amphiregulin and epiregulin expression in KRAS wild type colorectal primaries predicts response and survival benefit after treatment with cetuximab and irinotecan for metastatic disease. ASCO Gastrointestinal Cancers Symposium. 2008:Abstr. 411

52. Baker JB, Dutta D, Watson T, et al. Evaluation of tumor gene expression and KRAS mutations in FFPE tumor tissue as predictors of response to cetuximab in metastatic colorectal cancer. J Clin Oncol. 2008; 26s:A3512

53. Jonker DJ, Karapetis C, Harbison C, et al. High epiregulin (EREG) gene expression plus K-ras wild-type (WT) status as predictors of cetuximab benefit in the treatment of advanced colorectal cancer (ACRC): Results from NCIC CTG CO.17-A phase III trial of cetuximab versus best supportive care (BSC). J Clin Oncol. 2009;26: Abstr. 4016. 
54. Adams R, Maughan T. Predicting response to epidermal growth factor receptor-targeted therapy in colorectal cancer. Expert Rev Anticancer Ther. 2007;7:503-518.

55. Benvenuti S, Sartore-Bianchi A, Di Nicolantonio F, et al. Oncogenic activation of the RAS/RAF signaling pathway impairs the response of metastatic colorectal cancers to anti-epidermal growth factor receptor antibody therapies. Cancer Res. 2007;67(6):2643-2648.

56. Amado RG, Wolf M, Peeters M, et al. Wild-type KRAS is required for panitumumab efficacy in patients with metastatic colorectal cancer. J Clin Oncol. 2008;26(10):1626-1634.

57. Freeman DJ, Juan T, Reiner M. Association of K-RAS mutational status and clinical outcomes in patients with metastatic colorectal cancer receiving panitumumab alone. Clin Colorectal Cancer. 2008;7(3):184-190.

58. Cohn AL, Smith DA, Neubauer MA, et al. Results from panitumumab (pmab) regimen evaluation in colorectal cancer to estimate primary response to treatment (PRECEPT): Second-line treatment with pmab and FOLFIRI by tumor KRAS status. J Clin Oncol. 2009;26:Abstr. 4067.

59. Kohne CH, Mineur L, Greil R, et al. Primary analysis of a phase II study (20060314) combining first-line panitumumab with FOLFIRI in the treatment of patients with metastatic colorectal cancer. $A S C O$ Gastro-Intestinal Symposium. Orlando, US: 2010.

60. Barault L, Veyrie N, Jooste V, et al. Mutations in the RAS-MAPK, PI(3) $\mathrm{K}$ (phosphatidylinositol-3-OH kinase) signaling network correlate with poor survival in a population-based series of colon cancers. Int J Cancer. 2008;122:2255-2259.

61. Tan YH, Liu Y, Eu KW, et al. Detection of B-RAF V600E mutation by pyrosequencing. Pathology. 2008;40:295-298.

62. Calistri D, Rengucci C, Seymour I, et al. Mutation analysis of p53, KRAS and B-RAF genes in colorectal cancer progression. $J$ Cell Physiol. 2005;204:484-488.

63. Di Nicolantonio F, Martini M, Molinari F, et al. Wild-type B-RAF is required for response to panitumumab or cetuximab in metastatic colorectal cancer. J Clin Oncol. 2008;26: $5705-5712$.

64. Vehlo S, Oliveira C, Ferreira A, et al. The prevalence of PIK3CA mutations in gastric and colon cancer. Eur J Cancer. 2005;41:1649-1654.

65. Samuels Y, Diaz LA Jr, Schmidt-Kittler O, et al. Mutant PIK3CA promotes cell growth and invasion of human cancer cells. Cancer Cell. 2005;7:561-573.

66. Blanco-Aparicio C, Renner O, Leal J, Carnero A. PTEN, more than the AKT pathway. Carcinogenesis. 2007;28:1379-1386.

67. Carnero A, Blanco-Aparicio C, Renner O, et al. The PTEN/PI3K/AKT signalling pathway in cancer, therapeutic implications. Curr Cancer Drug Targets. 2008;8:187-198.

68. Perrone F, Lampis A, Orsenigo M, et al. PI3KCA/PTEN deregulation contributes to impaired responses to cetuximab in metastatic colorectal cancer patients. Ann Oncol. 2009;20:84-90.

69. Loupakis F, Pollina L, Stasi I, et al. PTEN expression and KRAS mutations on primary tumors and metastases in the prediction of benefit from cetuximab plus irinotecan for patients with metastatic colorectal cancer. J Clin Oncol. 2009;27(16):2622-2629.

70. Bardier A, Golmard J, Domont J, et al. Coexpression of EGFR, pEGFR, VEGF, pVEGF, PTEN, pAKT and p21 in colorectal cancer patients can have IHC variability between metastases and primary tumors and for EGFR.targeted therapies, p21 and VEGF appear reliably as predictive factors of response. J Clin Oncol. 2008; 26s:A22074.

Core Evidence

\section{Publish your work in this journal}

Core Evidence is an international, peer-reviewed open-access journal evaluating the evidence underlying the potential place in therapy of drugs throughout their development lifecycle from preclinical to postlaunch. The focus of each review is to evaluate the case for a new drug or class in outcome terms in specific indications and patient groups.

Submit your manuscript here: http://www.dovepress.com/core-evidence-journal
71. Frattini M, Saletti P, Romagnani E, et al. PTEN loss of expression predicts cetuximab efficacy in metastatic colorectal cancer patients. Br J Cancer. 2007;97:1139-1145.

72. Sartore-Bianchi A, Martini M, Molinari F, et al. PIK3CA mutations in colorectal cancer are associated with clinical resistance to EGFRtargeted monoclonal antibodies. Cancer Res. 2009;69:1851-1857.

73. Finocchiaro G, Cappuzzo F, Janne PA, et al. EGFR, HER2 and Kras as predictive factors for cetuximab sensitivity in colorectal cancer. $J$ Clin Oncol. 2007;25(1):Abstr. 4021.

74. Scartozzi M, Bearzi I, Mandolesi A, et al. Correlation of insulin-like growth factor 1 (IGF-1) expression and clinical outcome in K-RAS wild-type colorectal cancer patients treated with cetuximab-irinotecan. J Clin Oncol. 2009;26:Abstr. 4017.

75. Wheeler DL, Huang S, Kruser TJ, et al. Mechanisms of acquired resistance to cetuximab: role of HER (ErbB) family members. Oncogene. 2008;27(28):3944-3956.

76. Zhang W, Gordon M, Press OA, et al. Cyclin D1 and epidermal growth factor polymorphisms associated with survival in patients with advanced colorectal cancer treated with cetuximab. Pharmacogenet Genomics. 2006;16(7):475-483.

77. Vallböhmer D, Zhang W, Gordon M, et al. Molecular determinants of cetuximab efficacy. J Clin Oncol. 2005;23(15):3536-3544

78. Van Cutsem E, Peeters M, Siena S, et al. Open-label phase III trial of panitumumab plus best supportive care compared with best supportive care alone in patients with chemotherapy-refractory metastatic colorectal cancer. J Clin Oncol. 2007;25(13):1658-1664.

79. Peeters M, Siena S, Van Cutsem E, et al. Association of progression-free survival, overall survival and patient-reported outcomes by skin toxicity and KRAS status in patients receiving panitumumab monotherapy. Cancer. 2009;115:1544-1554.

80. Berlin J, Van Cutsem E, Peeters M, et al. Predictive value of skin toxicity severity for response to panitumumab in patients with metastatic colorectal cancer (mCRC): a pooled analysis of five clinical trials. $J$ Clin Oncol. 2007;25(18S):Abstr. 4134.

81. Schrag D. The price tag on progress - chemotherapy for colorectal cancer. $N$ Engl J Med. 2004;351:317-319.

82. Paramore LC, Thomas SK, Knopf KB, et al. Estimating costs of care for patients with newly diagnosed metastatic colorectal cancer. Clin Colorectal Cancer. 2006;6:52-58.

83. Bracco A, Farrimond BJ, Fitzgibbon JW, et al. A model to demonstrate the comparative costs between panitumumab and cetuximab for third-line metastatic colorectal cancer patients in Italy. ISPOR Annual European Congress. 2008;Athens, Greece.

84. Tappenden P, Jones R, Paisley S, Carroll C. Systematic review and economic evaluation of bevacizumab and cetuximab for the treatment of metastatic colorectal cancer. Health Technol Assess. 2007;11:1-128.

85. Norum J. Cetuximab in the treatment of metastatic colorectal cancer: a model-based cost-effectiveness analysis. J Chemother. 2006; 18:532-537.

86. Van Cutsem E, Lang I, D'haens G, et al. KRAS status and efficacy in the first-line treatment of patients with metastatic colorectal cancer (mCRC) treated with FOLFIRI with or without cetuximab: the CRYSTAL experience. J Clin Oncol. 2008;26:Abstr. 2.

87. Mancl EE, Kolesar JM, Vermeulen LC. Clinical and economic value of screening for Kras mutations as predictors of response to epidermal growth factor receptor inhibitors. Am J Health Syst Pharm. 2009; 66(23):2105-2112.

\section{Dovepress}

The manuscript management system is completely online and includes a very quick and fair peer-review system, which is all easy to use. Visit http://www.dovepress.com/testimonials.php to read real quotes from published authors. 